\title{
Tailoring the deep brain stimulation indications in Parkinson's disease
}

\author{
Individualizando as indicações para estimulação cerebral profunda na doença de Parkinson
} Egberto Reis Barbosa', Rubens Gisbert Cury ${ }^{1}$

\author{
1 Universidade de São Paulo, \\ Faculdade de Medicina, \\ Departamento de Neurologia, \\ Centro de Distúrbios do Movimento, \\ São Paulo SP, Brasil. \\ Correspondence: \\ Egberto Reis Barbosa:Av. Dr. Enéas \\ de Carvalho Aguiar, 255 / sala 5084; \\ 05403-900 São Paulo SP, Brasil; \\ E-mail:egbertob@8415.com.br \\ Conflict of interest: \\ There is no conflict of interest to \\ declare. \\ Received 08 May 2018 \\ Accepted 15 May 2018 \\ (cc) BY
}

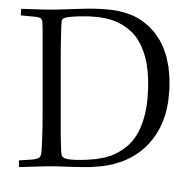

eep brain stimulation (DBS) has revolutionized the treatment of Parkinson's disease (PD) over the last 30 years and can be considered the "second honeymoon" in the subset of patients who undergo the surgery. Nevertheless, the dissemination of information on DBS in the scientific community and the general population is still suboptimal. Studies show that the expected number of patient candidates for DBS is higher than the actual number being referred for surgery, and one of the main reasons is poor knowledge of the selection criteria among the neurology community ${ }^{1}$. The surgery is not free of complications, and very careful selection of good candidates is a key component of successful therapy.

In this issue of the Journal, Brandão et al. ${ }^{2}$ highlight the importance of a large, multidisciplinary team of experts to assess potential PD patients as candidates for DBS, and the authors provide a comprehensive overview of current eligibility criteria for the surgery. The classical indications for DBS are based on motor symptoms (refractory motor complications or disabling tremor). However, a deeper evaluation of the baseline quality of life, the patient's expectations, and the intensity of nonmotor symptoms can be helpful in making better selections of good candidates and the most appropriate targets.

Recent papers have shown a high response rate in some nonmotor symptoms after DBS (especially pain and sleep), which may be a major determinant for improvement in quality of life ${ }^{3}$. Brandão et al. ${ }^{2}$ emphasize the importance of a neuropsychological assessment before surgery due to the high prevalence of impulse control disorders in PD patients referred for DBS. It is still controversial whether DBS could worsen or improve impulse control disorders. Reducing the dopaminergic medication load might improve behavioral features, but specific complications could be triggered by stimulating the limbic domain of the subthalamic nucleus. Recently, a secondary analysis of the EARLYSTIM trial (a study on subthalamic nucleus-DBS in PD with early motor complications) showed an improvement in hyperdopaminergic syndrome at two years after subthalamic nucleus-DBS, suggesting that the presence of hyperdopaminergic behaviors can be judged as additional arguments in favor of surgery ${ }^{4}$. On the other hand, it is well known that DBS can worsen depression and executive functions and, depending on the degree of these symptoms at baseline, the decision may trend against the surgery.

Besides the motor and nonmotor baseline features, predictive factors may help clinicians in making decisions. A good response to a levodopa challenge has been shown to be a reliable predictor of motor outcomes after DBS. Recently, structural and functional brain connectivity measured through tractography and functional MRI has been studied as a promising independent predictor of clinical outcome after DBS in $\mathrm{PD}^{5}$. Taken together, the levodopa response and the integrity of brain connectivity before surgery should provide more trustworthy information about the expected response to DBS and allow for a more "customized" indication.

Once defining a candidate for DBS, the pros and cons of the subthalamic nucleus or globus pallidus internus as targets should be balanced. Longer follow-up studies demonstrate that there is no "winner", but the fact that the number of studies with the subthalamic nucleus is higher than for the globus pallidus internus-DBS clearly demonstrates that the subthalamic nucleus has become the favored target across centers. Factors that should be considered in preoperative target assignment include the experience of the surgical center, the predominance of dyskinesias and on-period dystonia (which may favor the globus pallidus internus), and the need to reduce dopaminergic drug use due to side effects (which may favor the subthalamic nucleus). 
Challenges in the years to come involve the cognitive impairment that is seen in some PD patients, as well as gait disturbances that are not responsive to levodopa, which are symptoms that are usually not affected by the stimulation (or are even worsened). Attempts have been made to target the nucleus basalis of Meynert in the same trajectory as the globus pallidus internus in PD patients who have both cognitive and motor disturbances ${ }^{6}$. Pedunculopontine nucleus DBS and spinal cord stimulation have been associated with improvements in gait and freezing, but larger and longer studies are needed ${ }^{7,8}$.
The evolution in DBS therapy has been possible because of the uninterrupted work of multidisciplinary teams. In summary, an individualized decision based on motor and nonmotor symptoms, the baseline quality of life, and the patient's expectations should be the backbone of the multidisciplinary cooperation efforts to select the best candidates for DBS in PD. Greater knowledge of the inclusion criteria among the medical community could also increase the access to this underutilized therapy. Finally, a better understanding of biomarkers and circuitry dysfunctions in PD will be essential to neuromodulate new brain targets.

\section{References}

1. Lange M, Mauerer J, Schlaier J, Janzen A, Zeman F, Bogdahn U, et al. Underutilization of deep brain stimulation for Parkinson's disease? A survey on possible clinical reasons. Acta Neurochir (Wien). 2017 May;159(5):771-8. https://doi.org/10.1007/s00701-017-3122-3

2. Brandão P, Grippe TC, Modesto LC, Ferreira AGF, Silva FM, Pereira FF et al. Viewpoint: decisions about deep brain stimulation therapy in Parkinson's disease. Arq Neuropsiquiatr. 2018;76(6):411-20. https://doi.org/10.1590/0004-282X20180048

3. Cury RG, Galhardoni R, Fonoff ET, Dos Santos Ghilardi MG, Fonoff F, Arnaut D et al. Effects of deep brain stimulation on pain and other nonmotor symptoms in Parkinson disease. Neurology. 2014 Oct;83(16):1403-9. https://doi.org/10.1212/WNL.0000000000000887

4. Lhommée E, Wojtecki L, Czernecki V, Witt K, Maier F, Tonder L et al. Behavioural outcomes of subthalamic stimulation and medical therapy versus medical therapy alone for Parkinson's disease with early motor complications (EARLYSTIM trial): secondary analysis of an open-label randomised trial. Lancet Neurol. 2018;17(3):223-31. https://doi.org/10.1016/S1474-4422(18)30035-8

5. Horn A, Reich M, Vorwerk J, Li N, Wenzel G, Fang Q et al. Connectivity predicts deep brain stimulation outcome in Parkinson disease. Ann Neurol. 2017 Jul;82(1):67-78. https://doi.org/10.1002/ana.24974

6. Gratwicke J, Zrinzo L, Kahan J, Peters A, Beigi M, Akram H et al. Bilateral deep brain stimulation of the nucleus basalis of meynert for Parkinson disease dementia: a randomized clinical trial. JAMA Neurol. 2018 Feb;75(2):169-78. https://doi.org/10.1001/jamaneurol.2017.3762

7. Thevathasan W, Debu B, Aziz T, Bloem BR, Blahak C, Butson C et al. Pedunculopontine nucleus deep brain stimulation in Parkinson's disease: A clinical review. Mov Disord. 2018 Jan;33(1):10-20. https://doi.org/10.1002/mds.27098

8. Souza CP, Hamani C, Souza CO, Lopez Contreras WO, Ghilardi MGS, Cury RG et al. Spinal cord stimulation improves gait in patients with Parkinson's disease previously treated with deep brain stimulation. Mov Disord. 2017;32(2):278-82. https://doi.org/10.1002/mds.26850 